\title{
Studies on Combining Ability and Gene Action for Yield and Quality Traits in Hybrid Rice (Oryza sativa L.)
}

\author{
J. Buelah ${ }^{1 *}$, V. Ram reddy ${ }^{1}$, B. Srinivas ${ }^{2}$ and N. Balram ${ }^{3}$ \\ ${ }^{1}$ Department of Genetics and Plant Breeding, Agricultural College, PJTSAU, \\ Polasa, Jagtial, India \\ ${ }^{2}$ Plant Breeding, ${ }^{3}$ Plant Pathology, PJTSAU, Regional Agricultural Research Station, \\ Polasa, Jagtial, India \\ *Corresponding author
}

\section{A B S T R A C T}

Combining ability for yield and yield attributing traits in 24 hybrids generated by crossing four male sterile lines with six testers in line $\mathrm{x}$ tester fashion were studied along with their

\begin{tabular}{|l|}
\hline Keyw or d s \\
Hybrid Rice, \\
Oryza sativa
\end{tabular}
parents and checks (BIO 799 and PA 6129). The analysis revealed greater SCA variance than GCA variance for all the characters under study except for number of productive tillers per plant, hulling percentage, amylose content $(\%)$, gel consistency $(\mathrm{mm})$, alkali spreading value, kernel breadth $(\mathrm{mm})$, kernel length $(\mathrm{mm})$ and $\mathrm{L} / \mathrm{B}$ ratio which indicated the predominance of non-additive gene action in the inheritance of most of the traits and suggested for improvement through heterosis breeding. Among the parental lines, JMS 17B, CMS 14B, JGL 34990 and JMBR 44 were the best general combiners for grain yield and its contributing traits. Hence, these parents may be utilized in the development of superior hybrids. The hybrids CMS 14A X JGL 34985, JMS 18A X IRTON 270, JMS 17A X IRTON 270, CMS 14A X JGL 34990, JMS 18A X JGL 34450, JMS 13A X JGL 34450, JMS 13A X JMBR 44 and CMS 14A X JGL 35039 were the best specific combiners for grain yield and its contributing traits. Therefore, these promising hybrids based on per se performance, sca effects, gca effects of male and female parents could be recommended for heterosis breeding.

\section{Introduction}

Rice is a highly self-pollinated crop belongs to the gramineae family, producing edible starchy cereal grains and originated in South East Asia. The genus Oryza has two cultivated and 22 wild species. The cultivated species are Oryza sativa and Oryza glaberrima. Oryza sativa is grown all over the world and species glaberrima has been cultivated in few areas like West Africa. The global area under rice is 1.58 billion hectares with a production of 470.2 million tonnes per annum. While in India it is grown in about 43.79 million hectares with a production of 116.48 million tonnes and productivity of $2659 \mathrm{~kg} / \mathrm{ha}$. Whereas, in Telangana State it is grown in 19.32 lakh hectares with the production of 66.70 million tonnes and productivity of $3452 \quad \mathrm{~kg} / \mathrm{ha}$ 
(www.indiastat.com,2018-19).Rice is the leading food crop in the world, directly feeding nearly half of the world's population. Rice is the predominant food crop in India in terms of area, production and productivity. Rice plays an important role in ensuring food security and contributing to poverty and malnutrition alleviation.

To meet the demand of increasing population and to combat food security in India, the present yield levels needs to be increased up to 121 million tonnes by 2050 and the production of rice needs to be increased by almost two million tons every year. In India, population improvement rate is $1.04 \%$.

Till date High Yielding Varieties (HYV's) have satisfied rice demand but they have reached their saturated levels. To meet the demand of increasing population adoption of hybrid rice technology is an alternative. Since rice is a self-pollinated crop, hybrid seed production must be based on male sterility systems.

Success of Hybrid Rice Technology depends on efficient and economic seed production which demands the parental genotypes having efficacy to combine and give rise to improved genotypic combinations as the performance of a $F_{1}$ hybrid depends on choice of parents. Parents with favourable alleles on crossing could produce heterotic hybrids. Selection of parents with high GCA and hybrids with high SCA is a backbone for hybrid seed production in rice. The yield of $F_{1}$ hybrids will decrease by $0.8 \%$ when the seed purity decreases by $1 \%$, so it is very important to establish a sustainable system of seed production to ensure the purity of hybrid seeds in hybrid rice development.

Parental genotypes which are considered to be superior must prove to be a good donor and a good combiner for desirable traits.
Combining ability analysis (Sprague and Tatum, 1942) is one of the valuable tools available to ascertain the combining ability effects and helps in selecting the desirable parents and crosses in a large number of germplasm lines for the further exploitation. Combining ability is used in understanding the nature of gene action involved in the expression of quantitative traits and to predict the performance of the progenies.

The GCA is the average performance of a genotype in a series of hybrid combinations, whereas SCA refers to the performance of a combination of specific genotype in a particular cross. General combining ability of parents provides useful information on their selection and specific combining ability for better performance of hybrids besides elucidating the nature and magnitude of gene action in the inheritance of a particular character.

The high gca effect for a particular trait of a parent indicates the additive gene effect for the trait governed by the genes in the parent concerned. The GCA and SCA variances provide an estimation for additive and nonadditive gene actions, respectively (Falconer, 1967). Keeping this in view, the present investigation was carried out to study the combining ability and gene action in order to identify good combiners and superior hybrid combinations.

\section{Materials and Methods}

The present investigation was undertaken during Rabi, 2018-19 (crossing programme) and Kharif, 2019 (evaluation) at Regional Agricultural Research Station (RARS), Polasa, Jagtial of Telangana state and the experimental material used for the present experiment comprised of four lines viz., JMS 17A, JMS 13A, JMS 18A, CMS 14A, six restorer lines viz., JGL 35039, JGL 34450, 
JGL 34985, JGL 34990, JMBR 44 and IRTON 270 and their 24 hybrids produced by crossing lines and testers in Line $\mathrm{X}$ tester fashion along with two hybrid checks (BIO799 and PA 6129). Four lines and six testers were planted in a crossing block with a spacing of $20 \mathrm{x} 15 \mathrm{~cm}$ and crossing programme is carried out in a four $\mathrm{x}$ six, Line $\mathrm{x}$ tester mating design to produce 24 hybrids during Rabi, 2018-19.

Hybridization and clipping method was followed to obtain hybrids and during Kharif, 2019, 30 days old seedlings of 36 entries (four lines, six testers, 24 hybrids and 2 checks) were transplanted in the main field in Randomized Block Design in two replications. Each entry was planted in two rows of four meters length with a spacing of $20 \times 15 \mathrm{~cm}$ in two replications.

Five sample plants were randomly selected from each entry excluding the border plants to minimize error due to the border effect and the following data were recorded: Days to $50 \%$ flowering, plant height $(\mathrm{cm})$, panicle length $(\mathrm{cm})$,number of productive tillers per plant, number of grains per panicle, spikelet fertility (\%), 1000-grain weight (g), grain yield per plant $(\mathrm{g})$,hulling percentage, milling percentage, head rice recovery (\%), kernel length $(\mathrm{mm})$, kernel breadth $(\mathrm{mm}), \mathrm{L} / \mathrm{B}$ ratio, amylose content (\%), gelatinization temperature $\left({ }^{\mathrm{O}} \mathrm{C}\right)$, gel consistency $(\mathrm{mm})$ and alkali spreading value. Collected data were subjected to statistical analysis using line $x$ tester analysis by Kempthorne (1957).

\section{Results and Discussion}

\section{General and specific combining ability}

The analysis of variance for combining ability of all the traits under study is presented in the (Table 1) which showed that the variance due to treatments, parents and crosses was found significant for all the characters except for kernel length (mm), kernel breadth ( $\mathrm{mm})$ and $\mathrm{L} / \mathrm{B}$ ratio.

The variance due to parents $v s$ crosses was found to be significant for all the traits under study except for plant height $(\mathrm{cm})$, panicle length $(\mathrm{cm})$, 1000-grain weight $(\mathrm{g})$, kernel length $(\mathrm{mm})$, kernel breadth $(\mathrm{mm}), \mathrm{L} / \mathrm{B}$ ratio and alkali spreading value. The variance due to lines was found significant for all the traits except for panicle length $(\mathrm{cm})$, number of productive tillers per plant, kernel length $(\mathrm{mm})$, kernel breadth $(\mathrm{mm}), \mathrm{L} / \mathrm{B}$ ratio and alkali spreading value.

Whereas variance due to testers was found to be non-significant for kernel length $(\mathrm{mm})$, kernel breadth $(\mathrm{mm})$ and $\mathrm{L} / \mathrm{B}$ ratio and significant for all other characters. When the effects of parents were partitioned into lines, testers and line $\times$ tester effects, the interaction effects (lines $\times$ testers) were found to be significant for all the traits except for kernel length $(\mathrm{mm})$, kernel breadth $(\mathrm{mm})$ and L/B ratio under study.

This suggest that sufficient variability is available in the material under study.Similar works have been reported by Hasan et al., (2015) for parent vs crosses and testers, Archana Devi et al., (2017) for parents, crosses, parents $v s$ crosses, lines and line $\mathrm{x}$ tester interaction, Elshenawy et al., (2018) for lines, Amitkumar et al., (2019) for parents and parents $v s$ crosses and Bano and Singh (2019) for parents $v s$ crosses.

The results revealed that none of the parents showed significant gca effects for all the traits. Positive significant gca and sca effect is desirable for all the traits under study except for days to 50 per cent flowering and plant height where negative significant gca and sca effect is desirable. Character wise estimation of $\mathrm{gca}$ effects is presented in the (Table 2). 
Table.1 Analysis of variance for combining ability (Line x Tester) for yield and quality traits in rice

\begin{tabular}{|c|c|c|c|c|c|c|c|c|c|c|}
\hline Source of Variations & df & $\begin{array}{c}\text { Days to } 50 \% \\
\text { flowering }\end{array}$ & $\begin{array}{l}\text { Plant height } \\
\text { (cm) }\end{array}$ & $\begin{array}{c}\text { Panicle } \\
\text { length } \\
\text { (cm) }\end{array}$ & $\begin{array}{c}\text { No. of } \\
\text { productive } \\
\text { tillers per plant }\end{array}$ & $\begin{array}{l}\text { No. of grains per } \\
\text { panicle }\end{array}$ & $\begin{array}{c}\text { Spikelet } \\
\text { fertility } \\
(\%)\end{array}$ & $\begin{array}{l}1000 \text {-grain } \\
\text { weight } \\
\text { (g) }\end{array}$ & $\begin{array}{c}\text { Grain yield } \\
\text { per plant } \\
(\mathrm{g})\end{array}$ & $\begin{array}{c}\text { Hulling } \\
(\%)\end{array}$ \\
\hline Replicates & 1 & 0.75 & $16.45^{* *}$ & $4.20^{*}$ & 0.19 & $72.52 * *$ & 0.00 & 0.14 & 0.35 & $28.99 * *$ \\
\hline Treatments & 33 & $25.86^{* *}$ & $49.94 * *$ & $6.30 * *$ & $6.53 * *$ & $8022.18 * *$ & $100.23 * *$ & $29.20 * *$ & $96.96 * *$ & $42.43 * *$ \\
\hline Parents & 9 & $10.76^{* *}$ & $51.10 * *$ & $7.24 * *$ & $6.94 * *$ & $12116.53 * *$ & $28.89 * *$ & $49.47 * *$ & $87.80 * *$ & $7.27 * *$ \\
\hline Parents(Lines) & 3 & $15.17 * *$ & $64.97 * *$ & 1.81 & 2.13 & $3371.46^{* *}$ & $18.33 * *$ & $17.30 * *$ & $60.70 * *$ & $8.27 * *$ \\
\hline Parents(Tester) & 5 & $9.93 * *$ & $47.77 * *$ & $10.94 * *$ & $11.13 * *$ & $19666.28 * *$ & $40.33 * *$ & $70.26 * *$ & $119.74 * *$ & $8.12 * *$ \\
\hline Parents(L vsT) & 1 & 1.63 & $26.13 * *$ & $5.00 *$ & 0.41 & $603.01 * *$ & 3.33 & $41.98 * *$ & $9.41 * *$ & 0.00 \\
\hline Crosses & 23 & $29.95 * *$ & $51.60 * *$ & $6.18 * *$ & $6.06^{* *}$ & $6761.14 * *$ & $114.21 * *$ & $22.45 * *$ & $102.90 * *$ & $57.32 * *$ \\
\hline Line Effect & 3 & $25.81 * *$ & $16.58 * *$ & $3.32 *$ & $10.85 * *$ & $2628.69 * *$ & $257.25 * *$ & $4.38 *$ & $64.62 * *$ & $83.22 * *$ \\
\hline Tester Effect & 5 & $13.78 * *$ & $93.29 * *$ & $6.86^{* *}$ & $10.74 * *$ & $13167.34 * *$ & $110.33^{* *}$ & $33.35 * *$ & $125.72 * *$ & $161.99 * *$ \\
\hline Line x TesterEff. & 15 & $36.17 * *$ & $44.70 * *$ & $6.53 * *$ & $3.54 * *$ & $5452.24 * *$ & $86.90 * *$ & $22.43 * *$ & $102.95 * *$ & $17.25 * *$ \\
\hline Error & 33 & 0.96 & 4.17 & 1.31 & 0.48 & 214.49 & 4.45 & 0.78 & 2.44 & 3.79 \\
\hline Total & 37 & 13.23 & 26.93 & 3.81 & 3.46 & 4058.30 & 51.56 & 14.78 & 48.96 & 23.18 \\
\hline
\end{tabular}

Table.1 (cont.)

\begin{tabular}{|c|c|c|c|c|c|c|c|c|c|c|}
\hline Source of Variations & Df & $\begin{array}{l}\text { Milling } \\
(\%)\end{array}$ & $\begin{array}{l}\text { Head rice } \\
\text { recovery } \\
(\%)\end{array}$ & $\begin{array}{l}\text { Kernel } \\
\text { length } \\
(\mathrm{mm})\end{array}$ & $\begin{array}{c}\text { Kernel } \\
\text { breadth } \\
(\mathbf{m m})\end{array}$ & $\begin{array}{l}\mathrm{L} / \mathrm{B} \\
\text { ratio }\end{array}$ & $\begin{array}{c}\text { Amylose } \\
\text { content }(\%)\end{array}$ & $\begin{array}{c}\text { Gelatinization } \\
\text { Temperature } \\
\left({ }^{0} \mathrm{C}\right)\end{array}$ & $\begin{array}{c}\text { Gel consisteny } \\
(\mathrm{mm})\end{array}$ & $\begin{array}{c}\text { Alkali } \\
\text { spreading } \\
\text { value }\end{array}$ \\
\hline Replicates & 1 & $15.50 * *$ & 0.52 & 0.10 & 0.01 & 0.01 & 0.00 & 0.33 & $10.08 * *$ & 0.00 \\
\hline Treatments & 33 & $58.08 * *$ & $182.76^{* *}$ & 0.38 & 0.06 & 0.05 & $20.72 * *$ & $49.72 * *$ & $600.94 * *$ & $2.01 *$ \\
\hline Parents & 9 & $13.73 * *$ & $143.64 * *$ & 00.71 & 0.03 & 0.08 & $24.19 * *$ & $48.47 * *$ & $667.27 * *$ & $2.27 *$ \\
\hline Parents(Lines) & 3 & $9.19 * *$ & $85.74 * *$ & 00.10 & 0.03 & 0.02 & $25.99 * *$ & $38.46 * *$ & $513.33 * *$ & 2.13 \\
\hline Parents(Testers) & 5 & $19.16^{* *}$ & $195.29 * *$ & 00.98 & 0.04 & 0.11 & $27.77 * *$ & $63.68 * *$ & $892.55^{* *}$ & $2.73^{*}$ \\
\hline Parents(L vs T) & 1 & 0.21 & $59.07 * *$ & 11.15 & 0.00 & 0.06 & 0.88 & 2.41 & 2.70 & 0.41 \\
\hline Parents vsCrosses & 1 & $25.12 * *$ & $23.08 * *$ & 00.02 & 0.16 & 0.10 & $25.70 * *$ & $15.56 * *$ & $538.31 * *$ & 0.12 \\
\hline Crosses & 23 & $76.86 * *$ & $205.01 * *$ & 00.27 & 0.07 & 0.04 & $19.15 * *$ & $51.70 * *$ & $577.71 * *$ & $2.00 *$ \\
\hline Line Effect & 3 & $25.51 * *$ & $189.90 * *$ & 00.36 & 0.14 & 0.05 & $7.15 * *$ & $30.39 * *$ & $532.03 * *$ & 1.42 \\
\hline Tester Effect & 5 & $52.08 * *$ & $176.14 * *$ & 00.40 & 0.05 & 0.05 & $66.09 * *$ & $105.85^{* *}$ & $1860.70 * *$ & $5.28 * *$ \\
\hline Line $x$ Tester Eff. & 15 & $95.40 * *$ & $217.66^{* *}$ & 00.20 & 0.06 & 0.04 & $5.90 * *$ & $37.91 * *$ & $159.18 * *$ & 1.02 \\
\hline Error & 33 & 4.64 & 9.43 & 00.09 & 0.02 & 0.02 & 0.04 & 0.26 & 1.80 & 0.01 \\
\hline Total & 37 & 31.16 & 94.67 & 00.23 & 0.04 & 0.04 & 10.22 & 24.62 & 297.08 & 1.00 \\
\hline
\end{tabular}

*Significant at 5 per cent level**Significant at 1 percent level 
Table.2 Estimates of general combining ability $(g c a)$ effects for lines and testers for yield and quality traits in rice

\begin{tabular}{|c|c|c|c|c|c|c|c|c|c|c|c|c|c|c|c|c|c|c|}
\hline Source & $\begin{array}{c}\text { Days } \\
\text { to } \\
\mathbf{5 0 \%} \\
\text { floweri } \\
\text { ng }\end{array}$ & $\begin{array}{c}\text { Plant } \\
\text { Height } \\
\text { (cm) }\end{array}$ & $\begin{array}{c}\text { Panicl } \\
\text { e } \\
\text { length } \\
(\mathrm{cm})\end{array}$ & $\begin{array}{c}\text { No. of } \\
\text { produc } \\
\text { tive } \\
\text { tillers } \\
\text { per } \\
\text { plant }\end{array}$ & $\begin{array}{c}\text { No. of } \\
\text { grains } \\
\text { per } \\
\text { panicle }\end{array}$ & $\begin{array}{c}\text { Spikel } \\
\text { et } \\
\text { fertilit } \\
y \\
(\%)\end{array}$ & $\begin{array}{c}\mathbf{1 0 0 0} \\
\text { grain } \\
\text { weight } \\
\text { (g) }\end{array}$ & $\begin{array}{l}\text { Grain } \\
\text { yield per } \\
\text { plant } \\
(\mathrm{g})\end{array}$ & $\begin{array}{c}\text { Hullin } \\
\mathbf{g} \\
(\%)\end{array}$ & $\begin{array}{c}\text { Milli } \\
\mathbf{n g} \\
(\%)\end{array}$ & $\begin{array}{c}\text { Head } \\
\text { rice } \\
\text { recovery } \\
(\%)\end{array}$ & $\begin{array}{l}\text { Kernel } \\
\text { length } \\
(\mathbf{m m})\end{array}$ & $\begin{array}{c}\text { Kernel } \\
\text { breadth } \\
(\mathbf{m m})\end{array}$ & L/B ratio & $\begin{array}{c}\text { Amylose } \\
\text { content } \\
(\%)\end{array}$ & $\begin{array}{l}\text { Gelatinizatio } \\
\text { n temperatue } \\
\left({ }^{\circ} \mathbf{C}\right)\end{array}$ & $\begin{array}{c}\text { Gel } \\
\text { consisten } \\
\mathbf{y}(\mathbf{m m})\end{array}$ & $\begin{array}{c}\text { Alkali } \\
\text { spreadin } \\
\text { g value }\end{array}$ \\
\hline \multicolumn{19}{|l|}{ Parents } \\
\hline Lines & & & & & & & & & & & & & & & & & & \\
\hline JMS 17B & $0 . \overline{-}$ & $\begin{array}{l}1.63 \\
*\end{array}$ & 0.24 & 0.23 & 3.98 & $5.54 * *$ & 0.50 & $1.83 * *$ & $2.40 * *$ & $\begin{array}{c}4.91 * \\
*\end{array}$ & -0.12 & $0.13^{*}$ & -0.08 & $0.81 * *$ & $-0.58 * *$ & $-7.04 * *$ & $-0.38 * *$ & $4.91 * *$ \\
\hline JMS 13B & $-\overline{-}$ & $\begin{array}{r}- \\
1.18\end{array}$ & 0.24 & $-0.94 * *$ & 3.31 & $5.21^{* * *}$ & $\begin{array}{r}- \\
0.48\end{array}$ & $-3.24 * *$ & ${ }^{-}-$ & $\begin{array}{c}- \\
4.78^{*} \\
*\end{array}$ & 0.11 & 0.05 & 0.00 & $-0.86 * *$ & $-0.50^{*}$ & $5.04 * *$ & $0.29 * *$ & $-4.78 * *$ \\
\hline JMS 18B & $-0.79 *$ & $0 . \overline{-}$ & 0.31 & $0.52 *$ & $\begin{array}{c}- \\
21.02 * \\
*\end{array}$ & $\begin{array}{l}1.63 \\
*\end{array}$ & $\begin{array}{l}0.54 \\
*\end{array}$ & -0.12 & 1.04 & 0.44 & -0.18 & $-0.12 *$ & -0.01 & $0.46^{* *}$ & $2.33 * *$ & $-4.29 * *$ & $-0.21 * *$ & 0.44 \\
\hline CMS14B & $2.13^{* *}$ & $\begin{array}{r}- \\
0.19\end{array}$ & $\begin{array}{c}- \\
0.79 *\end{array}$ & $1.23 * *$ & $13.73^{*}$ & $-1.96^{*}$ & $\stackrel{-}{0.57^{*}}$ & $1.52^{*}$ & 0.29 & -0.57 & $0.19^{*}$ & -0.05 & $0.09 *$ & $-0.41 * *$ & $-1.25^{* *}$ & $6.29 * *$ & $0.29 * *$ & -0.57 \\
\hline \multicolumn{19}{|l|}{ Testers } \\
\hline JGL 35039 & $1.04 *$ & $5.90^{* *}$ & $1.49^{*}$ & $-1.94 * *$ & $\begin{array}{c}- \\
26.06^{*} \\
*\end{array}$ & $1.71^{*}$ & $-0.85^{*}$ & $-7.02 * *$ & $\stackrel{-}{-} 87^{* *}$ & $\begin{array}{c}- \\
7.70^{*} \\
*\end{array}$ & -0.21 & 0.04 & $-0.15^{*}$ & $-3.70 * *$ & $-6.25 * *$ & $22.75^{* *}$ & $1.21 * *$ & $-7.70 * *$ \\
\hline JGL 34450 & $\begin{array}{r}- \\
0.21\end{array}$ & $2.32 *$ & 0.13 & $1.06 * *$ & $14.81^{*}$ & $4.83 * *$ & $\begin{array}{r}- \\
0.30\end{array}$ & $-1.53 *$ & $3.24 * *$ & 0.76 & -0.02 & $-0.10^{*}$ & 0.07 & $1.57 * *$ & $2.50^{* *}$ & $-9.25^{* *}$ & $-0.29 * *$ & 0.76 \\
\hline JGL 34985 & $1.67 * *$ & $2.00^{-}$ & 0.20 & 0.44 & $\begin{array}{c}40.56^{*} \\
*\end{array}$ & $\begin{array}{c}- \\
6.42 * *\end{array}$ & $-\overline{-}$ & 0.57 & 0.90 & $\begin{array}{c}6.19^{*} \\
*\end{array}$ & -0.15 & -0.08 & 0.04 & $-2.95 * *$ & $-1.50 * *$ & $12.38 * *$ & $0.71 * *$ & $6.19 * *$ \\
\hline JGL 34990 & 0.17 & 0.45 & 0.34 & $-0.81^{*}$ & $\begin{array}{c}49.69^{*} \\
*\end{array}$ & 0.96 & $2.31 * *$ & $3.81 * *$ & 0.62 & $3.02 *$ & -0.16 & -0.02 & -0.04 & $2.47 * *$ & $2.88^{* *}$ & $-13.13^{* *}$ & $-0.79 * *$ & $3.02 *$ \\
\hline JMBR 44 & $2.08^{-} *$ & $3.20 * *$ & $\begin{array}{l}1.38 \\
*\end{array}$ & 0.31 & $\begin{array}{c}- \\
32.56^{*} \\
*\end{array}$ & 0.54 & $2.10 * *$ & $3.41 * *$ & $3.19 * *$ & -0.69 & 0.19 & 0.06 & 0.02 & $-0.57 * *$ & $-0.75^{* *}$ & $2.38 * *$ & -0.04 & -0.69 \\
\hline IRTON 270 & $\begin{array}{r}- \\
0.58\end{array}$ & 1.93 & 0.13 & $0.94 * *$ & $\begin{array}{c}- \\
46.44 * \\
*\end{array}$ & $\begin{array}{r}- \\
0.54\end{array}$ & $2.84 * *$ & 0.75 & 0.92 & -1.57 & $0.35^{*}$ & $0.10^{*}$ & 0.06 & $3.18 * *$ & $3.13 * *$ & $-15.13^{* *}$ & $-0.79 * *$ & -1.57 \\
\hline $\begin{array}{c}\text { CD95\% } \\
\text { GCA(Line) }\end{array}$ & 0.58 & 1.22 & 0.68 & 0.41 & $75^{8 .}$ & 1.26 & 0.53 & 0.93 & 1.16 & 1.83 & 0.18 & 0.08 & 0.08 & 0.11 & 0.30 & 0.80 & 0.07 & 1.83 \\
\hline $\begin{array}{c}\text { CD 95\% } \\
\text { GCA(Tester) }\end{array}$ & 0.72 & 1.49 & 0.84 & 0.51 & $\begin{array}{l}10.7 \\
1\end{array}$ & 1.54 & 0.64 & 1.14 & 1.42 & 2.25 & 0.22 & 0.09 & 0.10 & 0.14 & 0.37 & 0.98 & 0.09 & 2.25 \\
\hline
\end{tabular}


Table.3 Estimates of specific combining ability (sca) effects for yield and quality traits in rice

\begin{tabular}{|c|c|c|c|c|c|c|c|c|c|c|c|c|c|c|c|c|c|c|c|}
\hline $\begin{array}{c}\text { S.N } \\
0\end{array}$ & Crosses & $\begin{array}{c}\text { Days to } \\
50 \% \\
\text { flowering }\end{array}$ & $\begin{array}{c}\text { Plant } \\
\text { Height } \\
(\mathrm{cm})\end{array}$ & $\begin{array}{c}\text { Panicl } \\
\text { e } \\
\text { length } \\
(\mathrm{cm})\end{array}$ & $\begin{array}{c}\text { No. of } \\
\text { producti } \\
\text { ve tillers } \\
\text { per plant }\end{array}$ & $\begin{array}{c}\text { No. of } \\
\text { grains } \\
\text { per } \\
\text { panicle }\end{array}$ & $\begin{array}{c}\text { Spikelet } \\
\text { fertility } \\
(\%)\end{array}$ & $\begin{array}{l}\text { 1000-grain } \\
\text { weight (g) }\end{array}$ & $\begin{array}{l}\text { Grain yield } \\
\text { per plant } \\
\text { (g) }\end{array}$ & $\begin{array}{c}\text { Hulling } \\
(\%)\end{array}$ & $\underset{(\%)}{\text { Milling }}$ & $\begin{array}{c}\text { Head } \\
\text { rice } \\
\text { recovery } \\
(\%) \\
\end{array}$ & $\begin{array}{l}\text { Kernel } \\
\text { length } \\
(\mathbf{m m})\end{array}$ & $\begin{array}{c}\begin{array}{c}\text { Kernel } \\
\text { breadth } \\
(\mathrm{mm})\end{array} \\
\end{array}$ & $\begin{array}{c}\mathbf{L} / \mathbf{B} \\
\text { ratio }\end{array}$ & $\begin{array}{c}\text { Amylose } \\
\text { content } \\
(\%)\end{array}$ & $\begin{array}{c}\text { Gelatinization } \\
\text { temperatue } \\
\left({ }^{\circ} \mathrm{C}\right)\end{array}$ & $\begin{array}{c}\text { Gel } \\
\text { consistency } \\
(\mathbf{m m})\end{array}$ & $\begin{array}{c}\text { Alkali } \\
\text { spreading } \\
\text { value }\end{array}$ \\
\hline 1 & JMS 17 A $\times$ JGL 35039 & -0.04 & $-5.95 * *$ & 0.99 & -0.73 & $25.15^{*}$ & -2.79 & $2.58^{* *}$ & $-2.82^{*}$ & $4.87^{*}$ & $4.51^{*}$ & 1.84 & 0.24 & $0.29^{*}$ & -0.08 & $-0.44 * *$ & $2.08^{* *}$ & $5.67 * *$ & 0.13 \\
\hline 2 & JMS 17 A $\times$ JGL 34450 & $3.21 * *$ & 2.44 & -0.28 & $2.27^{* * *}$ & -7.23 & $6.58 * *$ & $-3.39^{* * *}$ & -1.06 & -1.24 & 1.07 & 4.21 & -0.10 & $-0.21 *$ & 0.19 & $1.55^{* * *}$ & -0.67 & $-7.83^{* * *}$ & $-0.38^{* * *}$ \\
\hline 3 & JMS 17 A J JGL 34985 & $2.33^{*}$ & $-5.00^{*}$ & 0.05 & $-2.10^{* *}$ & $30.52 *$ & $-5.17^{*}$ & $-1.36^{*}$ & -1.46 & $-2.90^{*}$ & -2.26 & 2.05 & -0.24 & $-0.30^{*}$ & 0.13 & $-1.04 * *$ & $-3.67 * *$ & $4.04 * *$ & $0.63 * *$ \\
\hline 4 & JMS 17 A $\times$ JGL 34990 & $-5.17 *$ & -1.00 & 0.34 & 0.15 & $-38.60^{*}$ & $3.46^{*}$ & $4.92 * *$ & -1.74 & 0.38 & $-6.72 * *$ & $-8.54 * *$ & 0.16 & -0.01 & -0.07 & $-1.65^{* * *}$ & -0.04 & $6.54 * *$ & 0.13 \\
\hline 5 & JMS $17 \mathrm{~A} \times$ JMBR 44 & $5.08^{* * *}$ & $5.20^{*}$ & 0.03 & $1.02 *$ & 5.65 & -1.54 & $-1.37 *$ & -0.84 & -1.19 & $-6.57 * *$ & $-14.71 * *$ & -0.21 & -0.04 & -0.18 & $1.74 * *$ & $3.58 * *$ & -8.46 ** & $-0.63^{* * *}$ \\
\hline 6 & JMS $17 A \times$ IRTON270 & $-5.42 * *$ & $4.32 *$ & -1.13 & -0.60 & -15.48 & -0.54 & $-1.37 *$ & $7.92 * *$ & 0.08 & $9.98 * *$ & $15.16^{* * *}$ & 0.16 & $0.27 *$ & 0.01 & -0.16 & $-1.29 *$ & 0.04 & 0.13 \\
\hline 7 & JMS $13 \mathrm{~A} \times$ JGL 35039 & $3.88 * *$ & $3.41^{*}$ & $-2.66^{*}$ & $1.44 *$ & $44.81^{* *}$ & 1.46 & $-6.87 * *$ & 2.00 & $-6.41 * *$ & -3.02 & $-8.56 * *$ & 0.16 & -0.02 & 0.12 & $0.69 * *$ & $-6.50 * *$ & -1.92 & $0.46 * *$ \\
\hline 8 & JMS 13 A $\times$ JGL 34450 & $-2.88 * *$ & $6.34 * *$ & $2.13^{*}$ & -0.06 & $-88.56^{* * *}$ & $5.33^{*}$ & $3.49 * *$ & $4.81 * *$ & -0.36 & 0.03 & $7.09 *$ & 0.23 & 0.04 & 0.04 & 0.28 & $2.25 * *$ & $-4.42 * *$ & -0.04 \\
\hline 9 & JMS 13 A $\times$ JGL 34985 & $1.75^{*}$ & -1.19 & -0.85 & -0.94 & $21.69 *$ & $-6.92 * *$ & $1.71^{*}$ & $-4.39^{* * *}$ & 1.28 & 0.71 & 0.07 & -0.01 & 0.06 & -0.09 & $0.94 * *$ & $1.25 *$ & -1.54 & -0.04 \\
\hline 10 & JMS 13A× JGL 34990 & $1.75^{*}$ & -2.54 & -0.31 & -0.19 & 15.56 & 0.71 & -0.99 & -0.08 & 0.60 & 2.06 & -1.17 & 0.01 & 0.06 & -0.09 & $1.18^{* *}$ & -0.13 & $-6.54 * *$ & $-0.54 * *$ \\
\hline 11 & JMS $13 \mathrm{~A} \times$ JMBR 44 & $-7.00 * *$ & $-4.09 *$ & 0.33 & 0.19 & 19.81 & -2.29 & $2.54 * *$ & $3.37^{*}$ & $3.54^{*}$ & -0.41 & 2.98 & 0.34 & -0.04 & $0.23^{*}$ & $-1.69 * *$ & $3.50 * *$ & $9.96 * *$ & $-0.29 *$ \\
\hline 12 & JMS 13A× IRTON270 & $2.50^{*}$ & -1.92 & 1.38 & -0.44 & -13.31 & 1.71 & 0.12 & $-5.72 * *$ & 1.35 & 0.62 & -0.40 & $-0.72 *$ & -0.09 & $-0.21 *$ & $-1.39 * *$ & -0.38 & $4.46 * *$ & $0.46 * *$ \\
\hline 13 & JMS $18 \mathrm{~A} \times$ JGL 35039 & $-3.46 * *$ & -0.67 & 0.51 & 0.02 & $-49.85^{* *}$ & -1.38 & $5.58 * *$ & $-3.87 *$ & -1.78 & $-12.81^{* * *}$ & $-10.88^{* * *}$ & -0.36 & -0.14 & 0.05 & $-0.74 * *$ & $-0.83^{*}$ & 0.42 & -0.04 \\
\hline 14 & JMS 18 A $\times$ JGL 34450 & -1.21 & $-5.88 * *$ & -0.60 & -0.98 & $29.27^{*}$ & -3.00 & $-1.64 *$ & $6.64 * *$ & 0.32 & 1.89 & -0.68 & -0.26 & 0.09 & -0.10 & $-1.20 * *$ & $-1.58 * *$ & $6.92 * *$ & $0.46 * *$ \\
\hline 15 & JMS 18A× JGL 34985 & $2.42^{*}$ & 1.98 & -1.63 & $1.65^{*}$ & $-72.48 * *$ & 1.75 & -0.65 & -5.11 ** & 1.36 & 3.10 & $5.76^{*}$ & 0.05 & 0.13 & -0.08 & 0.26 & $2.42 * *$ & $-2.21 *$ & $-0.54 * *$ \\
\hline 16 & JMS 18 A $\times$ JGL 34990 & $2.92 * *$ & -0.32 & -1.39 & $-1.10^{*}$ & -11.10 & $-6.63 * *$ & $-2.53 * * *$ & $-4.94 * *$ & 1.89 & 1.01 & 3.24 & 0.12 & 0.02 & 0.11 & $-1.45^{* *}$ & $-2.96^{* * *}$ & $10.29 * *$ & $0.96 * *$ \\
\hline 17 & JMS $18 \mathrm{~A} \times$ JMBR 44 & -1.33 & 1.78 & $2.60^{*}$ & -0.23 & $40.15 * *$ & -0.13 & -0.31 & -1.44 & -0.23 & $7.29 * *$ & 3.71 & 0.09 & 0.11 & -0.02 & $2.89^{* * *}$ & $3.67 * *$ & $-15.71 * *$ & $-0.79^{* * *}$ \\
\hline 18 & JMS 18Ax IRTON270 & 0.67 & $3.11^{*}$ & 0.50 & 0.65 & $64.02 * *$ & $9.38 * *$ & -0.44 & $8.72 * *$ & -1.57 & -0.48 & -1.15 & 0.36 & $-0.20^{*}$ & 0.03 & 0.24 & -0.71 & 0.29 & -0.04 \\
\hline 19 & CMS 14 A $\times$ JGL35039 & -0.38 & $3.22 *$ & 1.16 & -0.73 & -20.10 & 2.71 & -1.28 & $4.69 * *$ & $3.32^{*}$ & $11.31 * *$ & $17.61^{* * *}$ & -0.04 & -0.12 & -0.09 & $0.49^{*}$ & $5.25 * *$ & $-4.17 * *$ & $-0.54 * *$ \\
\hline 20 & CMS 14 A $\times$ JGL34450 & 0.88 & -2.90 & -1.25 & $-1.23^{*}$ & $66.52^{* * *}$ & $-8.92^{* * *}$ & $1.54^{*}$ & $-10.40^{* * *}$ & 1.27 & -3.00 & $-10.62 * *$ & 0.13 & 0.09 & -0.13 & $-0.63 * *$ & 0.00 & $5.33 * *$ & -0.04 \\
\hline 21 & CMS 14A× JGL34985 & $-6.50 * *$ & $4.22 *$ & $2.43^{*}$ & $1.40^{*}$ & 20.27 & $10.33^{* *}$ & 0.31 & $10.95 * *$ & 0.26 & -1.55 & $-7.87 *$ & 0.21 & 0.11 & 0.03 & -0.16 & 0.00 & -0.29 & -0.04 \\
\hline 22 & CMS 14 A $\times$ JGL34990 & 0.50 & $3.87 *$ & 1.36 & $1.15^{*}$ & $34.15^{*}$ & 2.46 & $-1.40 *$ & $6.77 * *$ & $-2.87 *$ & $3.65^{*}$ & $6.48^{*}$ & -0.28 & -0.07 & 0.04 & $1.93 * *$ & $3.13 * *$ & $-10.29 * *$ & $-0.54 * *$ \\
\hline 23 & CMS 14 A $\times$ JMBR 44 & $3.25 * *$ & -2.89 & $-2.95^{*}$ & -0.98 & $-65.60^{* * *}$ & $3.96^{*}$ & -0.86 & -1.09 & -2.13 & -0.30 & $8.02 *$ & -0.22 & -0.03 & -0.03 & $-2.94 * *$ & $-10.75 * *$ & $14.21 * *$ & $1.71 * *$ \\
\hline \multirow[t]{2}{*}{24} & CMS 14 AxIRTON 270 & $2.25 *$ & $-5.51 * *$ & -0.75 & 0.40 & $-35.23 *$ & $-10.54 * *$ & $1.70^{*}$ & $-10.92^{* * *}$ & 0.14 & $-10.11 * *$ & $-13.61 * *$ & 0.20 & 0.02 & 0.17 & $1.31 * *$ & $2.38 * *$ & -4.79 ** & $-0.54 * *$ \\
\hline & CD $95 \%$ SCA & 1.43 & 2.99 & 1.67 & 1.01 & 21.42 & 3.09 & 1.29 & 2.29 & 2.85 & 3.15 & 4.49 & 0.44 & 0.18 & 0.20 & 0.28 & 0.74 & 1.96 & 0.18 \\
\hline
\end{tabular}

Table.4 Estimates of general and specific combining ability variance, proportionate gene action and degree of dominance in rice

\begin{tabular}{|c|c|c|c|c|c|c|c|c|c|c|c|c|c|c|c|c|c|c|}
\hline $\begin{array}{l}\text { Source of } \\
\text { variation }\end{array}$ & $\begin{array}{c}\text { Days to } \\
50 \% \\
\text { flowering }\end{array}$ & $\begin{array}{c}\text { Plant } \\
\text { height } \\
(\mathrm{cm})\end{array}$ & $\begin{array}{c}\text { Panicle } \\
\text { length } \\
(\mathrm{cm})\end{array}$ & $\begin{array}{c}\text { No. of } \\
\text { productive } \\
\text { tillers }\end{array}$ & $\begin{array}{c}\text { No. of } \\
\text { grains } \\
\text { per } \\
\text { panicle }\end{array}$ & $\begin{array}{c}\text { Spikelet } \\
\text { fertility } \\
(\%)\end{array}$ & $\begin{array}{c}1000 \\
\text { grain } \\
\text { weight } \\
\text { (g) }\end{array}$ & $\begin{array}{l}\text { Grain } \\
\text { yield } \\
\text { per } \\
\text { plant } \\
(\text { g) }\end{array}$ & $\begin{array}{c}\text { Hulling } \\
(\%)\end{array}$ & $\begin{array}{c}\text { Milling } \\
(\%)\end{array}$ & $\begin{array}{l}\text { Head } \\
\text { rice } \\
\text { recovery } \\
(\%)\end{array}$ & $\begin{array}{l}\text { Kernel } \\
\text { length } \\
(\mathbf{m m})\end{array}$ & $\begin{array}{c}\text { Kernel } \\
\text { breadth } \\
(\mathbf{m m})\end{array}$ & $\begin{array}{l}\mathrm{L} / \mathrm{B} \\
\text { ratio }\end{array}$ & $\begin{array}{c}\text { Amylose } \\
\text { content } \\
(\%)\end{array}$ & $\begin{array}{c}\text { Gelatinization } \\
\text { temperature } \\
\left({ }^{\circ} \mathrm{C}\right)\end{array}$ & $\begin{array}{c}\text { Gel } \\
\text { consistency } \\
(\mathbf{m m})\end{array}$ & $\begin{array}{c}\text { Alkali } \\
\text { spreading } \\
\text { value }\end{array}$ \\
\hline$\sigma^{2}$ gca & 3.77 & 10.15 & 0.76 & 2.06 & 1536.71 & 35.87 & 3.62 & 18.55 & 23.76 & 6.83 & 34.72 & 0.06 & 0.02 & 0.01 & 7.32 & 13.57 & 238.91 & 0.67 \\
\hline$\sigma^{2}$ sca & 17.61 & 20.27 & 2.61 & 1.53 & 2618.88 & 41.22 & 10.83 & 50.25 & 6.73 & 45.38 & 104.12 & 0.06 & 0.02 & 0.01 & 2.93 & 18.82 & 78.69 & 0.50 \\
\hline$\sigma^{2}$ gca / $\sigma^{2}$ sca & 0.21 & 0.50 & 0.29 & 1.35 & 0.59 & 0.87 & 0.33 & 0.37 & 3.53 & 0.15 & 0.33 & 1.06 & 0.71 & 0.63 & 2.49 & 0.72 & 3.04 & 1.33 \\
\hline $\begin{array}{c}\text { Degree of } \\
\text { Dominance } \\
\sqrt{ } \sigma^{2} \text { sca } \\
\sqrt{ } \sigma^{2} \text { gca }\end{array}$ & 2.16 & 1.41 & 1.86 & 0.86 & 1.31 & 1.07 & 1.73 & 1.65 & 0.53 & 2.58 & 1.73 & 0.97 & 1.19 & 1.26 & 0.63 & 1.18 & 0.57 & 0.87 \\
\hline
\end{tabular}


Results revealed that the line 'JMS 17B' to be a good general combiner of grain yield per plant $(\mathrm{g})$ and some of its contributing traits viz., spikelet fertility (\%), hulling (\%), milling $(\%)$, kernel length $(\mathrm{mm}), \mathrm{L} / \mathrm{B}$ ratio and alkali spreading value, the line 'CMS 14B' to be a good general combiner of grain yield per plant (g) and some of its contributing traits viz.,.

Number of productive tillers per plant, number of grains per panicle, head rice recovery $(\%)$, kernel breadth, gelatinization temperature $\left({ }^{\circ} \mathrm{C}\right)$ and gel consistency $(\mathrm{mm})$, the tester 'JGL 34990' to be a good general combiner of grain yield per plant $(\mathrm{g})$ and some of its contributing traits viz..

Number of grains per panicle, milling (\%), $\mathrm{L} / \mathrm{B}$ ratio, amylose content $(\%)$ and alkali spreading value and the tester 'JMBR 44' to be a good general combiner of grain yield per plant $(\mathrm{g})$ and some of its contributing traits $v i z$,days to $50 \%$ flowering, panicle length (cm), 1000-grain weight (g), hulling (\%) and gelatinization temperature $\left({ }^{\circ} \mathrm{C}\right)$. Hence, these good general combiners may be extensively used in future for hybrid rice breeding programme.

Character wise estimation of sca effects is presented in the (Table 3 ) and results revealed that cross 'CMS 14A X JGL 34985 ' showed highest $s c a$ effect for grain yield per plant (g) and contributing characters like days to $50 \%$ flowering, panicle length $(\mathrm{cm})$, number of productive tillers per plant and spikelet fertility (\%) followed by the cross 'JMS 18A $\mathrm{X}$ IRTON 270' for grain yield per plant (g) and for other contributing traits i.e., number of grains per panicle and spikelet fertility (\%), the cross 'JMS 17A X IRTON 270' for grain yield per plant $(\mathrm{g})$ and for other contributing traits i.e., days to $50 \%$ flowering, milling (\%), head rice recovery (\%) and kernel breadth (mm), the cross 'CMS 14A X JGL 34990' for grain yield per plant (g) and for other contributing traits i.e., number of grains per panicle, milling (\%), head rice recovery $(\%)$, amylose content (\%) and gelatinization temperature $\left({ }^{\circ} \mathrm{C}\right)$, cross 'JMS 18A X JGL 34450 ' for grain yield per plant (g) and for other contributing traits i.e., plant height $(\mathrm{cm})$, number of grains per panicle, gel consistency $(\mathrm{mm})$ and alkali spreading value, the cross 'JMS 13A X JGL 34450' for grain yield per plant $(\mathrm{g})$ and for other contributing traits i.e., days to $50 \%$ flowering, spikelet fertility (\%), panicle length $(\mathrm{cm}), 1000$-grain weight $(\mathrm{g})$, head rice recovery (\%) and gelatinization temperature, the cross 'JMS 13A X JMBR 44' for grain yield per plant ( $\mathrm{g}$ ) and for other contributing traits i.e., gelatinization temperature $\left({ }^{\circ} \mathrm{C}\right), \mathrm{L} / \mathrm{B}$ ratio, gel consistency $(\mathrm{mm})$, days to $50 \%$ flowering, plant height (cm), 1000-grain weight (g) and hulling (\%) and the cross 'CMS 14A X JGL 35039' for grain yield per plant ( $g$ ) and for other contributing traits i.e., hulling (\%), milling $(\%)$, head rice recovery (\%), amylose content $(\%)$ and gelatinization temperature $\left({ }^{\circ} \mathrm{C}\right)$.

The characters viz., days to $50 \%$ flowering, plant height $(\mathrm{cm})$, panicle length $(\mathrm{cm})$, no. of grains per panicle, 1000 grain weight $(\mathrm{g})$, grain yield per plant $(\mathrm{g})$, spikelet fertility $(\%)$, milling (\%), head rice recovery (\%) and gelatinization temperature $\left({ }^{\circ} \mathrm{C}\right)$ exhibited greater SCA variance than GCA variance which indicated the predominance of nonadditive gene action in the inheritance of above mentioned traits.

Therefore the hybrid breeding programme is more useful for the improvement of these traits. GCA variance is greater than SCA variance of some characters viz., number of productive tillers per plant, hulling percentage, amylose content (\%), gel consistency $(\mathrm{mm})$ and alkali spreading value which indicated the predominace of additive gene action in the inheritance of above 
mentioned traits. GCA variance and SCA variance are equal for the characters kernel breadth $(\mathrm{mm})$, kernel length $(\mathrm{mm})$ and L/B ratio (Table 4 ). Predominance of non additive gene action for grain yield and its components was also reported by many other workers for grain yield per plant $(\mathrm{g})$.

Similar results were reported by several workers for days to 50 per cent flowering (Jayasudha and Sharma, 2009 and Ghara et al., 2012), plant height $(\mathrm{cm})$ (Selvarajet al., 2011, Dorosti and Monajjem, 2014), number of grains per panicle (Satheesh Kumar et al., 2016 and Rumanti et al., 2017), grain yield per plant (g) (Arifulislam et al., 2015, Santha et al., 2017), 1000-grain weight (g) (Vanave et al., 2018), milling percentage (\%) (Thakare et al., 2013 and Sreenivas et al., 2014), head rice recovery (\%) (Naseer Mohammad et al., 2016 and Rukmini Devi et al., 2018) and amylose content (\%) (Maleki et al., 2014).

In conclusion the predominance of specific combining ability (SCA) variance for most of the characters suggested the predominance of non-additive or dominant and epistatic gene action.

Hence, based on the results obtained in the present study the phenomenon of heterosis can be inferred to be playing important role in determining seed yield in case of rice hybrids and can be exploited commercially.

Parents JMS 17B, CMS 14A, JGL 34990 and JMBR 44 were best general combiners for yield and yield attributing traits and hybridsCMS 14A X JGL 34985, JMS 18A X IRTON 270, JMS 17A X IRTON 270, CMS 14A X JGL 34990, JMS 18A X JGL 34450, JMS 13A X JGL 34450, JMS 13A X JMBR 44 and CMS 14A X JGL35039. Hence, these parents and hybrids can be further used in the hybrid rice breeding programme.

\section{References}

Amit Kumar, A., Singh, V.J., Bhowmick, P.K., Vinod, K.K., Seth, R., Nagarajan, M., Ellur, R.K., Bollinedi, H., Gopala Krishnan, S and Ashok Kumar Singh. 2019. Assessing the performance of hybrids developed using isocytoplasmic restorers and identification of promising combiners in rice. Indian Journal of Genetics. 79(3): 523-535.

Archana Devi., PreetiKumari., RanjanDwivedi., SaketDwivedi., Verma, O.P., Singh, P.K and Dwivedi, D.K. 2017. Studies on heterosis and combining ability in rice (Oryza sativa L.) for morphophysiological traits under normal and saline conditions. International Journal of Current Microbiology and Applied Sciences. 6(8): 1558-1571.

Ariful Islam, M.D., Khaleque Mian, M.A., Rasul, G., Khaliq, Q.A and Mannan Akanda M.A. 2015.Estimation of gene action and variance components of some reproductive traits of rice (Oryza sativa L.) through line $\mathrm{x}$ tester analysis. Journal of Rice Research. 3 (3): 1-9.

Bano, D.A and Singh, S.P. 2019. Combining ability studies for yield and quality traits in aromatic genotypes of rice (Oryza sativa L.). Electronic Journal of Plant Breeding. 10 (2): 341-352.

Dorosti, H and Monajjem, S. 2014. Gene action and combining ability for grain yield and yield related traits in rice (Oryza sativa L.). The Journal of Agricultural Sciences. 9(3): 100-108.

Elshenawy, M.M., Anis, G.B., Elgamal, W.H and Ramadan, E.A. 2018. Genetic basis of combining ability for various quantitative traits using CMS lines of rice (Oryzasativa L.). The Serbian Journal of Agricultural Sciences.67(2): 125-135.

Falconer, S.P. 1967. Introduction to quantitative genetics. New York: The Ronald Press Company.

Ghara, A.G., Nematzadeh, G., Bagheri, N., Ebrahimi, A and Oladi, M. 2012. Evaluationof general and specific combining ability in parental lines of hybrid rice. International Journal of 
Agriculture Research.2 (4): 455-460.

Hasan, M.J., Kulsum, M.U., Hossain, E., Manzur Hossain, M., Mustafizur Rahman, $M$ and Farhat Rahmat, N.M. 2015.Combining ability analysis for identifying elite parents for heterotic rice hybrid. Academia Journal of Agricultural Research.3(5): 70-75.

Indiastat.2018-19. Agriculture production.http: //www.indiastat.com.

Jayasudha, S and Sharma, D. 2009. Combining ability and gene action analysis for yield and its components in rice (Oryza sativa L.).Journal of Rice Research. 2: 105-110.

Kempthorne, O. 1957.An Introduction to Genetic Statistics. John Wiley and Sons Inc: New york.

Maleki, M., Fotokian, M.H., Kajouri, F.D., Nouri, M.Z and Agahi, K. 2014.Study of combining ability and gene action of cooking quality traits in rice (Oryza sativa L.) using line $x$ tester analysis. Journal of Biodiversity and Environmental Sciences. 4 (3): 220-226.

Naseer Mohammad., Surendra Singh., EkhlaqueAhmad andSalilTewari. 2016. Assessment of combining ability for productivity and quality traits in aromatic rice (Oryza sativa L). International Journal of Bio-resource and Stress Management. 7(4): 525-532.

Rukmini Devi, K., Satish Chandra, B., Lingaiah, N., Hari, $Y$ and Venkanna, V. 2017.Analysis of variability, correlation and path coefficient studies for yield andquality traits in rice (Oryza sativa L.). Agriculture Science Digest. 37 (1): 1-9.

Rumanti, I.A., Purwoko, B.S., Dewi, I.S., Aswidinnoor, H and Widyastuti, Y. 2017. Combining ability for yield and agronomic traits in hybrid rice derived from wild abortive, gambiaca and kalingacytoplasmic male sterile lines. SABRAO Journal of Breeding and Genetics. 49(1): 69-76.

Santha, S., Vaithilingam, R., Karthikeyan, A and Jayaraj, T. 2017.Combining ability analysis and gene action of grain quality traits in rice (Oryza sativa $\mathrm{L}$.) using line $\mathrm{x}$ tester analysis.Journal of Applied and Natural Science. 9(2): 1236-1255.

Satheesh Kumar, P., Saranan, K and Sabesan, T. 2016. Selection of superior genotypesin rice (Oryza sativaL.) through combining ability analysis. International Journal of Agriculture and Science. 12(1): 15-21.

Selvaraj, I., Nagarajan, P., Thiyagarajan., Bharathi, M and Rabindran, R. 2011. Genetic parameters of variability, correlation and pathcoefficient studies for grain yield and other yield attributes among rice blast disease resistant genotypes of rice (Oryza sativa L.). African Journal of Biotechnology. 10(17): 3322-3334.

Sprague, G.F and Tatum, L.A. 1942. General versus specific combining ability in single crosses in corn.Agronomy Journal.34: 923-932.

Sreenivas, G., Cheralu, C., Devi, K.R and Murthy, K.G. 2014. Combining ability analysis for grain quality traits in rice (Oryza sativa L.).Environment and Ecology. 33 (1): 86191.

Thakare, I.S., Patel, A.L and Mehta, A.M. 2013. Line $\times$ tester analysis using CMS system in rice (Oryza sativa L.). The Bioscan. 8(4): 1379-1381.

Vanave, P.B., Vaidya, G.B and Jadhav, B.D. 2018. Combining ability of rice genotypes under coastal saline conditions. Electronic Journal of Plant Breeding. 9(1): 116-123.

\section{How to cite this article:}

Buelah, J., V. Ram reddy, B. Srinivas and Balram, N. 2020. Studies on Combining Ability and Gene Action for Yield and Quality Traits in Hybrid Rice (Oryza sativa L.). Int.J.Curr.Microbiol.App.Sci. 9(12): 1282-1290. doi: https://doi.org/10.20546/ijcmas.2020.912.158 